\title{
Solitary chemosensory cells in the respiratory and vomeronasal epithelium of the human nose: a pilot study*
}

\author{
Thomas Braun, Brigitte Mack, Matthias F. Kramer \\ Department of Otorhinolaryngology, Head and Neck Surgery, Ludwig Maximilian University, \\ Munich, Germany
}

\begin{abstract}
SUMMARY Background: Recently, solitary chemosensory cells have been described in the respiratory and vomeronasal epithelium of the rodent nose. Expressing G-protein coupled receptors for sweet, umami and bitter taste transduction, these cells are thought to mediate trigeminal reflexes upon stimulation with chemical irritants. The present study analyzes human nasal mucosa for the presence of solitary chemosensory cells.

Methodology: In human tissue samples from respiratory mucosa and the vomeronasal organ, gene expression of taste receptors families was studied in five patients using the Affymetrix Human Gene 1.0 ST Array and immunohistochemistry with specific antibodies. Results: Immunohistochemistry revealed that solitary chemosensory cells expressing G-protein coupled receptors for sweet, umami and bitter taste transduction are present in the human nose. cDNA microarray analysis congruently showed that cells expressing bitter taste receptors accumulate in the vomeronasal organ compared to the respiratory epithelium.

Conclusions: Solitary chemosensory cells expressing taste receptors are also present in the human nose. Since they are thought to mediate trigeminal reflexes, their role in the pathogenesis of nasal hyperreagibility should be elucidated in further studies.
\end{abstract}

Key words: solitary chemosensory cells, taste receptors, human nose, vomeronasal organ

\section{INTRODUCTION}

Solitary chemosensory cells (SCC) were first described in the gill apparatus of aquatic vertebrates ${ }^{(1,2)}$. Recently, SCC were also found in the respiratory epithelium of rodents, where their distribution and physiology has been studied ${ }^{(3-7)}$. There is agreement upon the classification of SCC as a part of the trigeminal chemesthetic system, which also relies on free nerve endings ${ }^{(3)}$. In contrast to free nerve endings, SCC use taste receptors and their related downstream signaling cascade ${ }^{(8)}$ for the detection of environmental stimuli ${ }^{(6,7,9)}$. In the rhinopharynx of rats, T1R3, the subunit of both umami and sweet taste receptors (T1R gene family), is present ${ }^{(6)}$; furthermore, Tizzano et al., found evidence for the presence of eight bitter taste receptors (T2R gene family) in the same study (in humans, at least 25 different T2R genes coding for different bitter taste receptors are believed to be functionally active ${ }^{(8)}$ ). However, despite the expression of taste receptors, stimulation of SCC is not thought to lead to a sensation of taste, but irritation or pain due to the connection with trigeminal fibers ${ }^{(3)}$. In mice, SCC of the trachea have been reported to play a physiological role in the regulation of breathing (4), and nasal SCC residing densely at the entrance of the vomeronasal organ have been found to play a role in regulating the access of stimuli to this sensory organ ${ }^{(5)}$.

These recent animal studies revealed that SCC do have a physiological function in the respiratory system and are not only remnants of a primitive chemosensory system. Since our working group studies nasal diseases in humans, we speculated that SCC might also be connected to pathophysiological conditions of the nose. To our best knowledge, it remains unknown if SCC are also present in the human nose. Therefore, the present study was initiated to search for SCC in the human nasal mucosa. Since in mice, an accumulation of SCC expressing bitter taste receptors is described for the vomeronasal duct ${ }^{(5)}$, we analyzed not only tissue samples of respiratory mucosa, but also vomeronasal epithelium to be able to compare our results with the present animal studies. 


\section{MATERIALS AND METHODS}

The study was approved by the faculty's ethics commission (project number 021-09).

\section{Tissue samples}

Tissue samples were harvested from macroscopically identifiable vomeronasal duct in patients undergoing septoplasty for nasal obstruction who did not suffer from any other nasal diseases (Figure 1). Informed consent was obtained preoperatively from each individual participating. Additionally, tissue samples from septal respiratory nasal mucosa were harvested from the same patient. Tissue samples were instantly put into physiological sodium chloride solution and frozen at $-80^{\circ} \mathrm{C}$.

\section{Immunohistochemistry}

Shock-frozen, Tissue-Tek-embedded (Sakura Finetek Europe BV, Alphen aan den Rijn, Netherlands) serial sections (4 $\mu \mathrm{m})$ from five cases of nasal tissue and two cases of healthy tongue (circumvallate papillae) were investigated. Primary antibodies used were rabbit anti-rat T1R1 and T1R2 (Alpha Diagnostics, San Antonio, TX, USA) and rabbit anti-human T2R4 (Novus Biologicals, Littleton, CO, USA), diluted 1:500 in phosphate buffered saline (PBS). Immunostaining was performed using the avidin-biotin-peroxidase complex method (Vectastain, Vector Laboratories, Burlingame, CA, USA), according to the manufacturer's protocol. Briefly, after fixation for ten minutes in acetone, followed by five minutes with $3.5 \%$ fresh paraformaldehyde, endogenous peroxidase was destroyed with $0.03 \%$ $\mathrm{H}_{2} \mathrm{O}_{2} / \mathrm{PBS}$ for ten minutes, and nonspecific antigenic sites were blocked with normal goat serum. Sections were then incubated with the respective primary antibody over night at $4{ }^{\circ} \mathrm{C}$, followed by incubation with biotinylated anti-rabbit immunoglobulins and subsequently with avidin-biotin-peroxidase complex for 30 minutes at room temperature for each step. After each step, sections were washed with PBS. Specific peroxidase activity was developed with $0.05 \%$ 3-amino-9-ethylcarbazol (Sigma, Deisenhofen, Germany) and $0.02 \% \mathrm{H}_{2} \mathrm{O}_{2} / 0.1 \mathrm{M}$ sodium acetate buffer ( $\mathrm{pH}$ 5.5). Counterstaining was done with Mayer's hematoxylin. Control stains were performed by omitting the specific antibody. Immunoreactive areas of serial sections from corresponding regions were evaluated by an investigator using a light microscope.

\section{cDNA microarray analysis}

To analyze mRNA for taste receptors present in respiratory and vomeronasal epithelium, a cDNA microarray analysis was used ${ }^{(10)}$. Frozen tissue samples were sent to the Competence Center for Fluorescent Bioanalytics of the University of Regensburg, Germany, where RNA extraction, cDNA synthesis and gene expression analysis using Human Gene 1.0 ST Array (Affymetrix, Santa Clara, CA, USA) was performed using internal protocols. Relative gene expression in the vomeronasal duct was analyzed in comparison to respiratory epithelium in tissue pairs of five patients by calculating log-2-transformation of the mean signal and also the mean fold change.

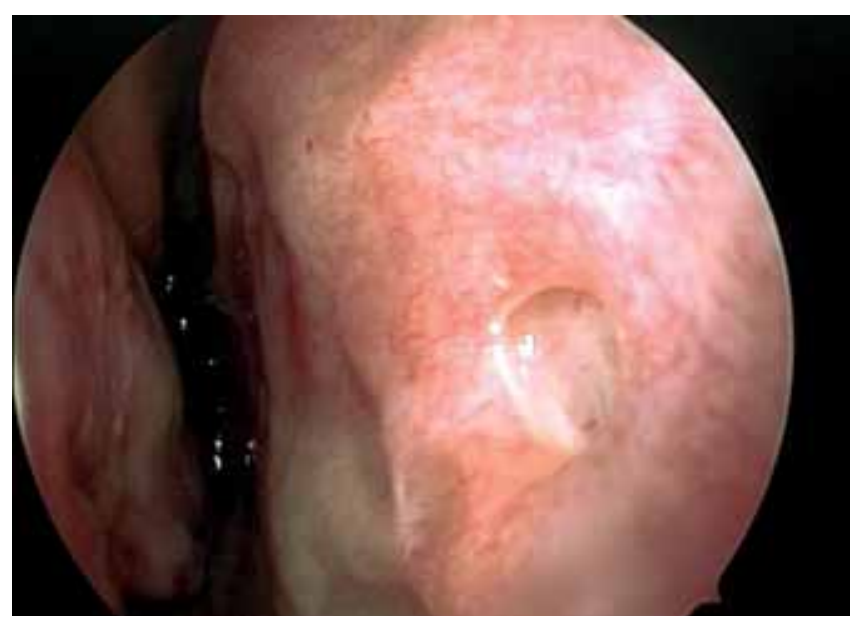

Figure 1. Vomeronasal organ (pit) in the right nasal septum of an adult male.

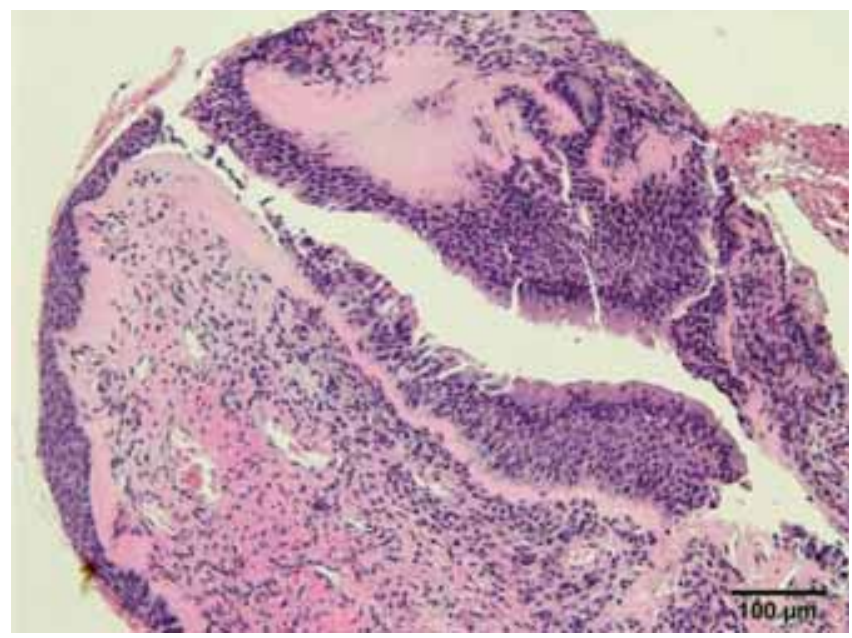

Figure 2. Longitudinally cut hematoxylin-eosin stained paraffin section revealing the vomeronasal duct with its characteristic epithelium (magnification: $100 \mathrm{x}$ ).

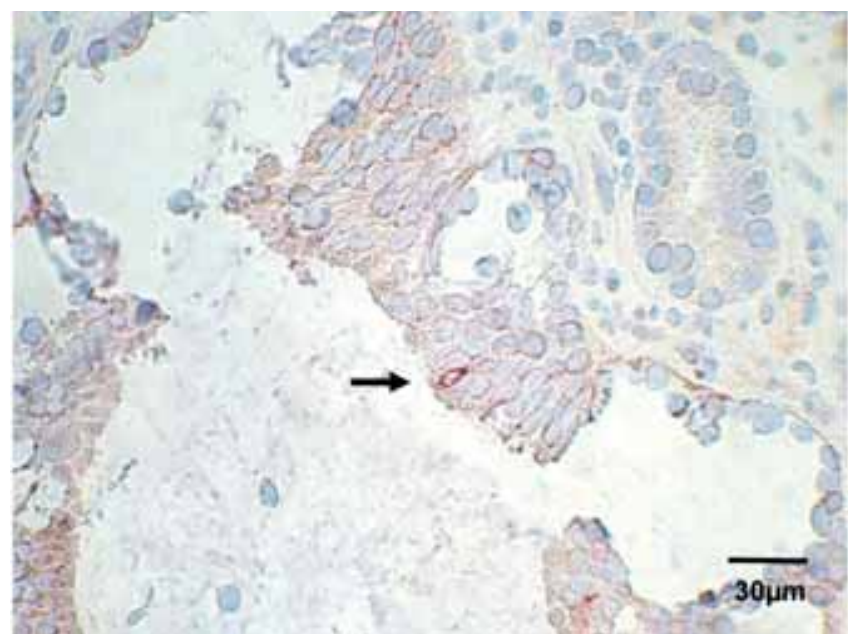

Figure 3. Solitary chemonsensory cell positive for T1R2 in the epithelium of the vomeronasal duct of a human nose (magnification: $400 \mathrm{x})$. 


\section{RESULTS}

To confirm expression of taste receptors in the human nasal epithelium, immunohistochemistry using specific antibodies for T1R1, T1R2 and T2R4 receptors was performed. As a positive control, taste buds of the human tongue were used; as expected, all receptor antibodies yielded a positive staining in human taste buds (not shown).

We found positive staining for T1R1 and T1R2 only on very few solitary cells. In 30 standard serial cuts of a particular case (five cases in total, representative examples shown in Figure 2-6) a maximum of only 1-2 positive cells were identified both remote from and in the proximity of the vomeronasal duct. As an example, Figure 3 shows a solitary cell positive for T1R2 in the epithelium of the vomeronasal duct. Figure 4 shows a solitary cell (or maybe two cells on adjacent sections) positive for both T1R1 (Figure 4a) and T1R2 (Figure 4b) in an excretory duct of a nasal gland. For both T1R1 and T1R2, only anti-rat antibodies were commercially available at the time of the study, which caused an increased unspecific background staining. However, the negative control (Figure 4c) shows that the solitary cell stained for T1R1 and T1R2 cannot be misinterpreted as unspecifically stained.

As an example to demonstrate expression of bitter taste receptors, we chose T2R4 since cDNA microarray analysis (discussed later in this section) suggested a quantitative relevance. Approximately 1-2 solitary cells positive for T2R4 were found in 30 standard serial cuts of the case investigated, remote from the vomeronasal duct (Figure 5). In contrast, a significantly larger population (approximately ten) of solitary cells positive for T2R4 was detected in the vomeronasal duct, as seen in Figure 6.

To objectify the distribution of taste receptor-expressing cells estimated by light microscopy and to yield additional evidence for the presence of taste receptors in the human nose also on the gene level, cDNA microarray analysis was performed. While we found no increased expression of the three T1 receptors (T1R1-3) in the human vomeronasal epithelium relative to respiratory nasal control tissue of the same patients, several T2 receptors covered by the Human Gene 1.0 ST Array showed a fold change of $\geq 2$ (T2R4, T2R13, T2R 14, T2R 31, T2R50; Table 1). No increased gene expression in the human vomeronasal organ was found for some established or putative intracellular elements of bitter taste signal transduction ${ }^{(8)}$, such as gustducin subunits beta and gamma (with subunit alpha, GNAT3, not covered by our cDNA microarray), the TRPM5 channel and phospholipase $\mathrm{C}$ beta2, while the expression of phospholipase $\mathrm{C}$ beta4 was increased (Table 1).

\section{DISCUSSION}

This is the first report about the presence of taste receptors ${ }^{(8)}$ in the human nose. cDNA microarray analysis and immunohistochemistry both showed that the human respiratory and vomeronasal epithelium contains taste receptors. We could describe solitary cells positive for T1R1, the specific subunit of
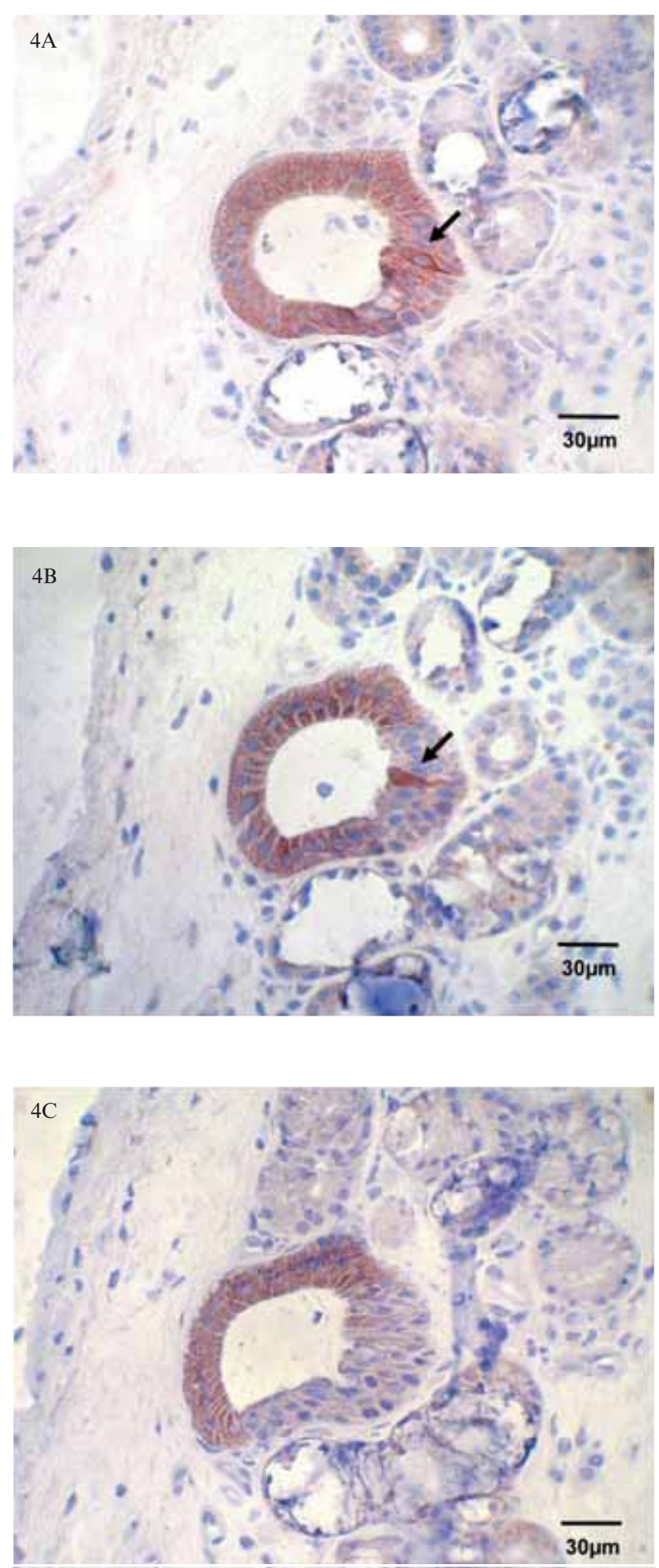

Figure 4. Solitary chemonsensory cell positive for both T1R1 (a) and T1R2 (b) in an excretory duct of a human nasal gland (magnification: $400 \mathrm{x})$. Negative control (c). 
Table 1. Results of the Affymetrix Human Gene 1.0 ST Array for tissue samples of adult humans from respiratory (R. E.) and vomeronasal epithelium (V. E.) in comparison.

\begin{tabular}{|c|c|c|c|c|}
\hline \multirow[t]{2}{*}{ Gene } & & \multicolumn{2}{|c|}{$\begin{array}{c}\text { Mean Signal } \\
\text { (log2-transformed) }\end{array}$} & \multirow[t]{2}{*}{ Fold Change } \\
\hline & & R. E. & V. E. & \\
\hline \multicolumn{5}{|c|}{ T1 Receptors } \\
\hline T1R1 & taste receptor, type 1, member 1 & 5.17 & 5.29 & 1.09 \\
\hline T1R2 & taste receptor, type 1, member 2 & 5.72 & 5.38 & -1.27 \\
\hline T1R3 & taste receptor, type 1, member 3 & 6.34 & 6.21 & -1.10 \\
\hline \multicolumn{5}{|c|}{ T2 Receptors } \\
\hline $\mathrm{T} 2 \mathrm{R} 1$ & taste receptor, type 2, member 1 & 3.91 & 4.01 & 1.07 \\
\hline $\mathrm{T} 2 \mathrm{R} 3$ & taste receptor, type 2, member 3 & 5.57 & 6.25 & 1.60 \\
\hline T2R4 & taste receptor, type 2 , member 4 & 5.68 & 7.09 & 2.65 \\
\hline T2R5 & taste receptor, type 2 , member 5 & 5.87 & 6.60 & 1.66 \\
\hline T2R7 & taste receptor, type 2, member 7 & 2.47 & 2.11 & -1.29 \\
\hline $\mathrm{T} 2 \mathrm{R} 8$ & taste receptor, type 2, member 8 & 2.74 & 2.83 & 1.07 \\
\hline T2R10 & taste receptor, type 2 , member 10 & 5.03 & 5.57 & 1.45 \\
\hline T2R13 & taste receptor, type 2, member 13 & 4.80 & 5.78 & 1.97 \\
\hline T2R14 & taste receptor, type 2 , member 14 & 4.84 & 6.01 & 2.25 \\
\hline T2R16 & taste receptor, type 2, member 16 & 2.78 & 2.68 & -1.07 \\
\hline T2R19 & taste receptor, type 2, member 19 & 5.48 & 6.02 & 1.45 \\
\hline $\mathrm{T} 2 \mathrm{R} 20$ & taste receptor, type 2, member 20 & 5.46 & 5.98 & 1.44 \\
\hline T2R31 & taste receptor, type 2 , member 31 & 5.42 & 6.43 & 2.01 \\
\hline T2R38 & taste receptor, type 2, member 38 & 4.48 & 4.51 & 1.02 \\
\hline T2R39 & taste receptor, type 2, member 39 & 3.04 & 3.20 & 1.12 \\
\hline $\mathrm{T} 2 \mathrm{R} 40$ & taste receptor, type 2, member 40 & 3.79 & 3.62 & -1.12 \\
\hline T2R41 & taste receptor, type 2, member 41 & 5.24 & 4.87 & -1.29 \\
\hline $\mathrm{T} 2 \mathrm{R} 42$ & taste receptor, type 2, member 42 & 3.78 & 3.72 & -1.04 \\
\hline T2R43 & taste receptor, type 2, member 43 & 3.36 & 4.30 & 1.92 \\
\hline T2R46 & taste receptor, type 2, member 46 & 3.83 & 4.26 & 1.35 \\
\hline T2R50 & taste receptor, type 2, member 50 & 3.80 & 4.80 & 2.00 \\
\hline T2R60 & taste receptor, type 2, member 60 & 4.97 & 4.27 & -1.62 \\
\hline \multicolumn{5}{|c|}{ Components of intracellular bitter taste signalling } \\
\hline GNB1 & guanine nucleotide binding protein, beta polypeptide 1 & 9.77 & 10.13 & 1.28 \\
\hline GNB3 & guanine nucleotide binding protein, beta polypeptide 3 & 5.83 & 5.78 & -1.03 \\
\hline GNG13 & guanine nucleotide binding protein, gamma 13 & 6.13 & 5.77 & -1.28 \\
\hline TRPM5 & transient receptor potential cation channel, subfamily M, member 5 & 6.40 & 5.92 & -1.40 \\
\hline PLCB1 & phospholipase $\mathrm{C}$, beta 1 & 6.23 & 7.53 & 2.46 \\
\hline PLCB2 & phospholipase $\mathrm{C}$, beta 2 & 5.87 & 6.00 & 1.09 \\
\hline PLCB3 & phospholipase $\mathrm{C}$, beta 3 & 7.33 & 6.78 & -1.46 \\
\hline PLCB4 & phospholipase $\mathrm{C}$, beta 4 & 6.50 & 8.16 & 3.15 \\
\hline
\end{tabular}

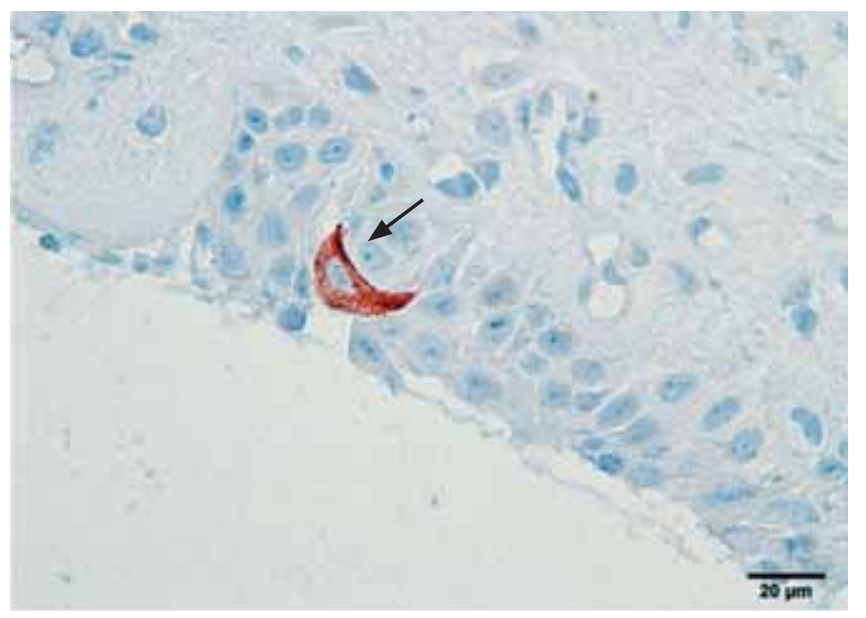

Figure 5. Solitary chemonsensory cell positive for T2R4 in the respiratory epithelium of a human nose (magnification: $400 \mathrm{x}$ ). a receptor heterodimer $(\mathrm{T} 1 \mathrm{R} 1+\mathrm{T} 1 \mathrm{R} 3)$ for umami taste, and also for T1R2, the specific subunit of the sweet taste receptor $(\mathrm{T} 1 \mathrm{R} 2+\mathrm{T} 1 \mathrm{R} 3)^{\left({ }^{8}\right)}$. Cells positive for the bitter taste receptor $\mathrm{T} 2 \mathrm{R} 4{ }^{(8)}$ were found to be present both in the respiratory and vomeronasal epithelium, but accumulate in the latter.

An expression of taste receptors in humans outside the tongue has been shown only for the gastrointestinal tract ${ }^{(11,12)}$, but not the nose or vomeronasal organ. Our results are in agreement with the experiments of Tizzano et al., (7) and Ogura et al., (5), who report that trigeminally innervated solitary chemosensory cells expressing taste receptors for umami, sweet and bitter tastants are present in the nose of mice, with bitter taste receptors accumulating in the proximity of the vomeronasal organ.

There are highly variable reports about the presence of a vomeronasal organ in adult humans (namely $25-100 \%$ ) ${ }^{(13)}$. In this study, we intentionally chose only subjects with a visible vome- 

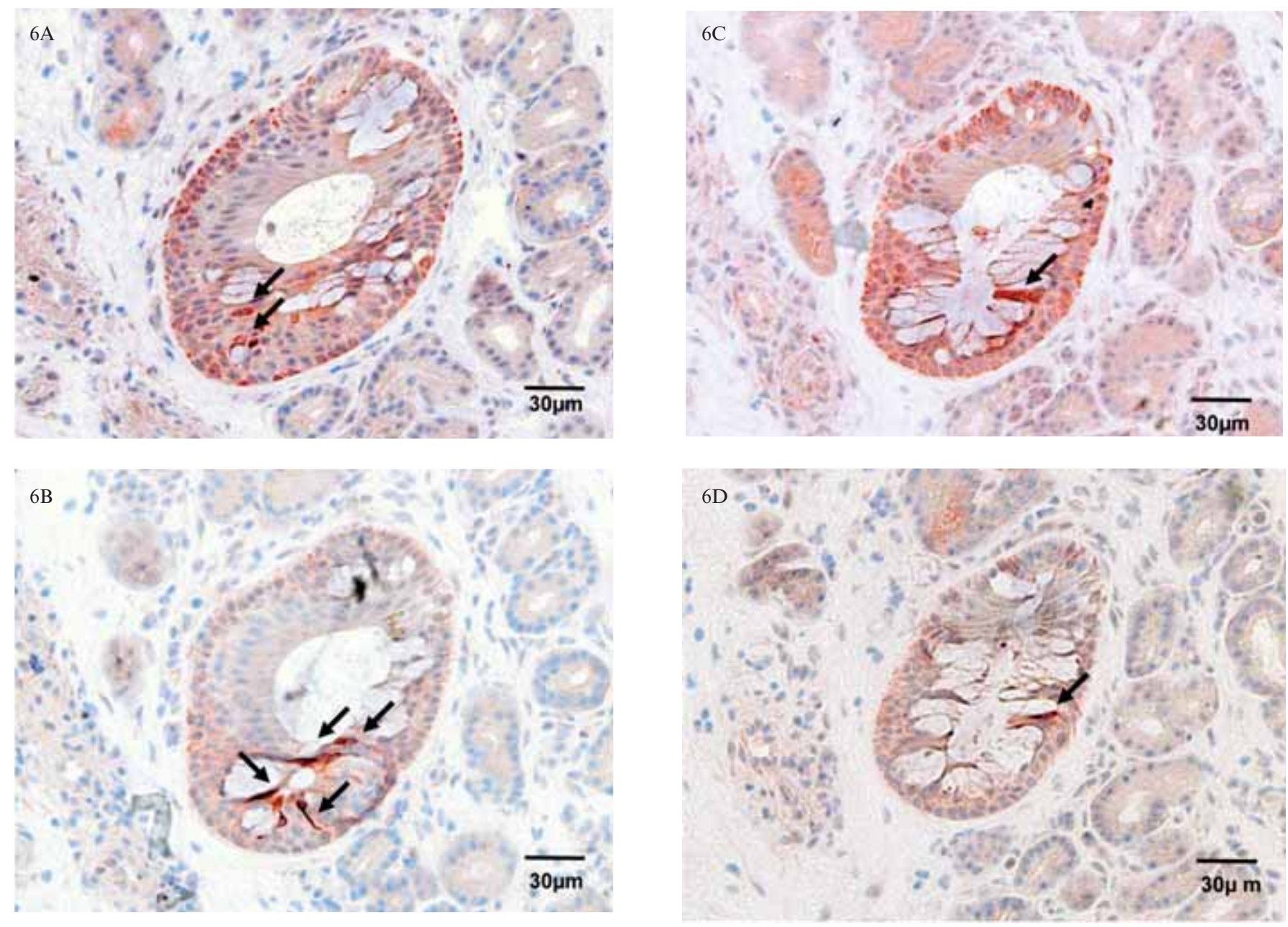

Figure 6. Serial cuts from a human vomeronasal duct demonstrating an accumulation of solitary chemonsensory cells positive for T2R4 (magnification: $400 \mathrm{x}$ ).

ronasal duct for harvesting tissue samples to allow comparison with the literature on rodents, which describes the finding that a considerable number of SCC expressing bitter taste receptors is located in the area of the vomeronasal organ ${ }^{(5)}$. Since our study in human subjects yielded analogous results, the approach of comparing respiratory and vomeronasal mucosa is important in the validation of our results. Because the vomeronasal organ in adult humans seems to degenerate to a highly variable extent (cf. e. g. ${ }^{(13,14)}$ ), it can be assumed that also SCC associated with this organ are present to a highly variable extent in adult humans. However, our study was not intended to specifically describe SCC associated with the vomeronasal organ or even speculate about the relevance in vomeronasal chemosensation, but to demonstrate the presence of SCC in human subjects.

Expression of taste receptors is thought to be a strongly characteristic marker of solitary chemosensory cells of rodent airways ${ }^{(6,7,9)}$. Since additional specific markers are not known, the cells described in the human tissue samples of this study cannot be proven to be the same kind of SCC as described in the literature. We did not perform immunohistochemical analysis of the known elements of downstream signaling for human taste sensors due to the lack of commercially available appropriate antibodies for use with human tissue at the time of our study. When comparing tissue samples from remote nasal control tissue with samples from the vomeronasal duct, where cells positive for the bitter taste receptor T2R 4 were found to accumulate, we did not find evidence for increased expression of the established downstream signaling elements in bitter taste transduction in the human tongue, TRPM5, gustducin or phospholipase C beta2 ${ }^{(15)}$, but expression of phospholipase $\mathrm{C}$ beta4, which was found in taste buds of the rat ${ }^{(16)}$, was increased.

The present study must be taken as a pilot study, suggesting the presence of SCC in the human nose. Further studies must be undertaken to elucidate if SCC of the human nose are also functionally active as it has been shown in the animal model. It is unlikely that SCC in the vomeronasal epithelium expressing bitter taste receptors have any impact on human chemosensation, since the vomeronasal organ of humans is thought to be functionally inactive by most experts ${ }^{(13)}$. In mice, SCC in the neighborhood of the vomeronasal organ are thought to regulate fluid access to the vomeronasal duct ${ }^{(5)}$. For humans, however, we think that SCC not associated with the vomero- 
nasal organ (if the latter is present at all) might be of clinical importance: Since SCC are thought to modulate trigeminal reflexes, they might play a role in nasal hyperreagibility, e. g. nasal pruritus and the urge to sneeze, and could therefore be involved in the pathophysiology of nasal disorders. Pharmacological antagonists for taste receptors are presently developed: GIV3727 inhibits bitter taste transduction ${ }^{(17)}$, while lactisole is an antagonist at the sweet taste receptor ${ }^{(18)}$. One could speculate that these substances might influence SCC of the human nose, which could lead to the development of a new kind of pharmaceuticals to treat nasal disorders. Future research using in vivo or in vitro stimulation with known ligands of the taste receptors expressed by SCC of the human nose will elucidate the possible functionality of these cells.

\section{ACKNOWLEDGEMENTS}

This work was supported by Friedrich-Baur-Stiftung (project number 41/10).

\section{AUTHORSHIP CONTRIBUTION}

TB: conceptual design, funding, tissue harvesting, coordination of DNA microarray analysis and immunohistochemistry, manuscript preparation; BM: immunohistochemistry, technical assistance; MFK: working group leader, proofreading of the manuscript.

\section{CONFLICT OF INTEREST}

All authors state that there is no conflict of interest.

\section{REFERENCES}

1. Kotrschal K. Solitary chemosensory cells: why do primary aquatic vertebrates need another taste system? Trends Ecol Evol. 1996; 11: 110-114.

2. Sbarbati A, Osculati F. Solitary chemosensory cells in mammals? Cells Tissues Organs. 2003; 175: 51-55.

3. Finger TE, Bottger B, Hansen A, Anderson KT, Alimohammadi H, Silver WL. Solitary chemoreceptor cells in the nasal cavity serve as sentinels of respiration. Proc Natl Acad Sci USA. 2003; 100: 89818986.

4. Krasteva G, Canning BJ, Hartmann P, et al. Cholinergic chemosensory cells in the trachea regulate breathing. Proc Natl Acad Sci USA. 2011.

5. Ogura T, Krosnowski K, Zhang L, Bekkerman M, Lin W. Chemoreception regulates chemical access to mouse vomeronasal organ: role of solitary chemosensory cells. PLoS One. 2010; 5: e11924.
6. Tizzano M, Cristofoletti M, Sbarbati A, Finger TE. Expression of taste receptors in solitary chemosensory cells of rodent airways. BMC Pulm Med. 2011; 11: 3.

7. Tizzano M, Gulbransen BD, Vandenbeuch A, et al. Nasal chemosensory cells use bitter taste signaling to detect irritants and bacterial signals. Proc Natl Acad Sci USA. 2010; 107: 3210-3215.

8. Drayna D. Human taste genetics. Annu Rev Genomics Hum Genet. 2005; 6: 217-235.

9. Gulbransen BD, Clapp TR, Finger TE, Kinnamon SC. Nasal solitary chemoreceptor cell responses to bitter and trigeminal stimulants in vitro. J Neurophysiol. 2008; 99: 2929-2937.

10. Brazma A, Vilo J. Gene expression data analysis. FEBS letters. 2000; 480: 17-24.

11. Behrens M, Meyerhof W. Oral and extraoral bitter taste receptors. Results Probl Cell Differ. 2010; 52: 87-99.

12. Bezencon C, le Coutre J, Damak S. Taste-signaling proteins are coexpressed in solitary intestinal epithelial cells. Chem Senses. 2007; 32: 41-49.

13. Knecht M, Witt M, Abolmaali N, Hüttenbrink KB, Hummel T. Das vomeronasale Organ des Menschen. Nervenarzt. 2003; 74: 858-862.

14. Brown JW. The nervus terminalis in insectivorous bat embryos and notes on its presence during human ontogeny. Ann NY Acad Sci. 1987; 519: 184-200.

15. Behrens M, Meyerhof W. Mammalian bitter taste perception. Results Probl Cell Differ. 2009; 47: 203-220.

16. Toyono T, Kataoka S, Seta Y, Toyoshima K. Expression of phospholipase C-beta4 in rat circumvallate taste buds. Chem Senses. 2005; 30 Suppl 1: i27-28.

17. Slack JP, Brockhoff A, Batram C et al. Modulation of bitter taste perception by a small molecule hTAS2R antagonist. Current biology. CB 2010; 20: 1104-1109.

18. Jiang P, Cui M, Zhao B, et al. Lactisole interacts with the transmembrane domains of human T1R3 to inhibit sweet taste. The Journal of biological chemistry 2005; 280: 15238-15246.

\section{Dr. med. Thomas Braun}

Klinik und Poliklinik für Hals-Nasen-Ohrenheilkunde der Ludwig-Maximilians-Universität München

Marchioninistraße 15

D-81377 München

Germany

Tel: +49-89-7095 0

Fax: +49-89-7095 6869

E-mail: thomas.braun@med.uni-muenchen.de 